Citation

Archived version

Published version

http://dx.doi.org/10.1007/s00134-015-3806-1

Journal homepage

http://link.springer.com/journal/134

Author contact

greet.vandenberghe@med.kuleuven.be

$+32(0) 16344021$

IR

Güiza F, Depreitere B, (2015), Intensive Care Med. 2015 Apr 18.
Visualizing the pressure and time burden of intracranial hypertension in adult and paediatric traumatic brain injury.

Author manuscript: the content is identical to the content of the published paper, but without the final typesetting by the publisher

https://lirias.kuleuven.be/handle/123456789/494906 


\title{
Visualizing the pressure and time burden of intracranial hypertension in adult and paediatric traumatic brain injury.
}

\author{
Fabian Güiza $\left({ }^{*}\right)$, Bart Depreitere $(*)$, Ian Piper, Giuseppe Citerio, lain Chambers, Patricia A. Jones, Tsz-Yan Milly \\ Lo, Per Enblad, Pelle Nillson, Bart Feyen, Philippe Jorens, Andrew Maas, Martin U Schuhmann, Rob Donald, \\ Laura Moss, Greet Van den Berghe, Geert Meyfroidt \\ $(*)$ Contributed equally.
}

The authors declare no conflicts of interest

Intensive Care Med

Received: 16 February 2015

Accepted: 5 April 2015

Published online: 18 April 2015

\section{Take-home message:}

In adult and paediatric patients suffering from severe traumatic brain injury, an approximately exponential curve describes the relationship between intensity and duration of episodes of increased intracranial pressure (ICP) and worse clinical outcomes. In children, compared to adults, this occurs at lower ICP thresholds of shorter duration.

\section{Electronic supplementary material}

The online version of this article (doi:10.1007/s00134-015-3806-1) contains supplementary material, which is available to authorized users.

F. Güiza , G. Van den Berghe, G. Meyfroidt (corresponding author)

Department of Intensive Care Medicine,

University Hospitals Leuven, Herestraat 49,

3000 Leuven, Belgium

e-mail: geert.meyfroidt@uzleuven.be

Tel.: +32 16344021

B. Depreitere

Department of Neurosurgery, University

Hospitals Leuven, Leuven, Belgium

I. Piper

Department of Clinical Physics, Southern

General Hospital, Glasgow, UK

G. Citerio

San Gerardo Hospital, Monza, Italy 
I. Chambers

James Cook University Hospital, Medical

Physics, Middlesbroughnza, UK

P. A. Jones

Department of Paediatric Neurology, Royal Hospital for Sick Children, Edinburgh, UK

T.-Y. M. Lo

Department of Paediatric Intensive Care, Royal Hospital for Sick Children,

Edinburgh, UK

P. Enblad, P. Nillson

Department of Neuroscience, Neurosurgery,

Uppsala, Sweden

B. Feyen , A. Maas

Department of Neurosurgery, Antwerp

University Hospital, Edegem, Belgium

P. Jorens

Department of Intensive Care Medicine, Antwerp University Hospital, Edegem, Belgium

M. U. Schuhmann

Klinik für Neurochirurgie,

Universitätsklinikum Tübingen, Tübingen,

Germany

R. Donald

School of Mathematics and Statistics,

University of Glasgow, Glasgow, UK

L. Moss

Department of Clinical Physics and

Bioengineering, NHS Greater Glasgow \&

Clyde, Glasgow, UK 


\section{Abstract:}

Purpose:

To assess the impact of the duration and intensity of episodes of increased intracranial pressure on 6-month neurological outcome in adult and paediatric traumatic brain injury.

Methods:

Analysis of prospectively collected minute-by-minute intracranial pressure and mean arterial blood pressure data of 261 adult and 99 paediatric traumatic brain injury patients from multiple European centres. The relationship of episodes of elevated intracranial pressure (defined as a pressure above a certain threshold during a certain time) with 6-month Glasgow Outcome Scale was visualized in a colour-coded plot.

Results:

The colour-coded plot illustrates the intuitive concept that episodes of higher intracranial pressure can only be tolerated for shorter durations: the curve that delineates the duration and intensity of those intracranial pressure episodes associated with worse outcome is an approximately exponential decay curve. In children, the curve resembles that of adults, but the delineation between episodes associated with worse outcome occurs at lower intracranial pressure thresholds. Intracranial pressures above $20 \mathrm{mmHg}$ lasting longer than 37 minutes in adults, and longer than 8 minutes in children, are associated with worse outcomes. In a multivariate model, together with known baseline risk factors for outcome in severe traumatic brain injury, the cumulative intracranial pressure-time burden is independently associated with mortality. When cerebrovascular autoregulation, assessed with the low-frequency autoregulation index, is impaired, the ability to tolerate elevated intracranial pressures is reduced. When the cerebral perfusion pressure is below $50 \mathrm{mmHg}$, all intracranial pressure insults, regardless of duration are associated with worse outcome.

\section{Conclusions:}

The intracranial pressure-time burden associated with worse outcome is visualised in a colour-coded plot. In children, secondary injury occurs at lower intracranial pressure thresholds as compared to adults. Impaired cerebrovascular autoregulation reduces the ability to tolerate intracranial pressure insults. Thus, $50 \mathrm{mmHg}$ might be the lower acceptable threshold for cerebral perfusion pressure.

\section{Key words:}

Traumatic brain injury - Adults - Children - Intracranial pressure - Cerebral perfusion pressure Cerebrovascular autoregulation 


\section{Introduction}

Traumatic brain injury (TBI) remains one of the most important health care problems worldwide [1], even when advancements in TBI management have led to improvements in outcome over the past decades [2-4]. Intracranial pressure (ICP) monitoring, first described in the 1950s [5], was introduced as a monitoring tool for severe $\mathrm{TBI}$ in the 1960s [6]. The most recent version of the Brain Trauma Foundation (BTF) guidelines recommends monitoring of ICP in every salvageable patient with severe TBI [7]. Although these guidelines acknowledge a lack of level I evidence, the recommended treatment threshold for ICP-lowering therapies is 20 $\mathrm{mmHg}$, based mainly on observational data [8-12]. An increased ICP is an independent risk factor for mortality $[13,14]$, and the total burden of ICP derangements, calculated as the area under the ICP curve (measured over time) above $20 \mathrm{mmHg}$, is an independent predictor of worse outcome in adults [15,16] and children [17]. Paediatric guidelines on ICP/cerebral perfusion pressure (CPP) management [18] have largely followed those for adults. An observational study of 81 children with TBI demonstrated that the ICP threshold associated with worse outcomes is lower, and differs in 3 age bands [17].

Aggressive ICP management is not free of risk [19]. In the Decompressive Craniectomy in Patients with Severe TBI (DECRA) trial [20], early decompressive craniectomy when ICP rose above $20 \mathrm{mmHg}$ resulted in a higher proportion of patients with poor neurological outcome as compared to standard care; however, after correction of baseline group imbalance (pupil reactivity), the outcomes were not different in the two groups. The multicentre randomized controlled BEST-TRIP trial (Benchmark Evidence from South American Trials: Treatment of Intracranial Pressure) [21] could not demonstrate the superiority of a TBI management strategy with ICP monitoring and treatment at $20 \mathrm{mmHg}$, versus a purely clinical and radiological management strategy. Notwithstanding these results, ICP monitoring is still considered to provide important information to detect and prevent secondary injury after severe TBI [22-24]. The BEST-TRIP trial has fostered a renewed interest in the definition of secondary insults and therapeutic thresholds for ICP and CPP.

The purpose of the present study is an exploratory analysis on prospective data from continuously monitored adult and paediatric TBI patients to assess the impact of duration and intensity of ICP insults, on 6 months neurological outcome. In addition, we will assess the impact of cerebrovascular autoregulation and CPP on the capacity to tolerate these insults. This research has been presented before, in part, at the 2014 International 
Symposium on Intensive Care and Emergency Medicine (ISICEM, Brussels) [25], and at the 2014 European Society of Intensive Care Medicine Congress (ESICM, Barcelona) [26].

\section{Materials and Methods}

Patients and data

The adult cohort was comprised of 261 TBI patients, aged 16 years and older.

- From the European Union Brain-IT project [27,28], 166 patients admitted to 22 neuro-ICUs in 11 European countries between March 2003 and July 2005 were included. The Multi-Centre Research Ethics Committee for Scotland (MREC/02/0/9) granted the use of these data for scientific purposes on 14 February 2002.

- Data of the remaining 95 adult patients were collected from the ICU of four centres: 38 patients from the San Gerardo Hospital Monza, Italy, between March 2010 and April 2013; 25 patients from the University Hospitals Leuven, Belgium, between September 2010 and September 2013; 20 patients from the University Hospital Edegem (Antwerp), Belgium, as part of the 'Individualized targeted monitoring in neurocritical care' (NEMO) project, between May 2010 and June 2013 [29]; and 12 patients from University Hospital Tübingen, Germany, between February and December 2009. All data referring to the identity of these patients were removed; ethical committee approval was obtained in all centres to use these data for analysis.

The paediatric cohort consisted of 99 TBI patients, aged between 2 and 16 years.

- 81 patients were part of a study on TBI in children, recruited during 62 non-consecutive months up to July 2003, from two paediatric centres in Edinburgh and Newcastle, UK [17]. The study had local ethics committee and management approval in both centres and informed consent was obtained before enrolment.

- The remaining 18 paediatric patients were part of the Brain-IT database.

Patients were managed according to BTF guidelines [30]. Data collection in both cohorts included baseline risk factors (age, gender, admission GCS, admission pupil reactivity), minute-by-minute ICP and mean arterial blood pressure (MAP) monitoring data, and Glasgow Outcome Score (GOS) at 6 months [Electronic Supplementary material (ESM) Table 1]. For the paediatric patients, a modified GOS was used, as described in the original paper [17]. Monitoring data in the NEMO database was recorded and stored every second; the median value of 
each minute interval was taken to obtain a minute-by-minute value. In all data sets, minute-by-minute signals were reviewed independently by two clinicians in Leuven (GM, BDP), and artefacts that were obvious through visual inspection of the time series were removed and not used for further analysis (ESM Table 10).

Visualization method:

The univariate association between ICP insults and GOS at 6 months was done using the visualization method explained in Fig. 1. First, each time series of minute-by-minute ICP data was segmented into different episodes, called 'ICP insults'. ICP insults were defined according to their intensity (I) and duration (D). Intensity thresholds from 10 to $40 \mathrm{mmHg}$ on the $\mathrm{X}$-axis and duration thresholds from 5 to $360 \mathrm{~min}$ on the $\mathrm{Y}$-axis were considered. For every ICP insult, the Pearson correlation coefficient between GOS and the average number of these types of ICP insults per patient in each GOS category was computed. The correlation coefficient ranges from -1 to 1 and measures the degree of linear dependency: a negative value indicates that the particular ICP insult occurs more frequently with lower GOS; a positive correlation indicates that the particular ICP insult is more frequent with higher GOS. A contour plot showing lines of equal correlation was derived from the grid and colour coded: correlations of -1 and +1 are shown in dark red and dark blue, respectively. The contour for zero correlation was highlighted in black, and defined as the 'transition curve', because it indicates the transition into the region of insult types that occur more frequently in patients with lower GOS.

Role of cerebrovascular autoregulation:

The low-frequency autoregulation index (LAx) is computed as a moving minute-by-minute correlation of previous values of ICP and MAP [31]. The LAx ranges in value from -1 to +1 and was calculated for every minute of the patients' ICU stay. This allowed one to compute the average autoregulatory state during each ICP insult. An insult was classified as 'active' if its average LAx was negative or zero, and 'passive' if the average LAx was positive. The visualization method described above was repeated considering active and passive episodes separately.

Role of CPP:

The association between ICP insults and outcome was visualized for three different thresholds of CPP: 50, 60, and $70 \mathrm{mmHg}$. 
Multivariate analysis:

In order to assess the independent association of the cumulative dose of ICP insults with outcome, multivariate logistic regression models for 6-month mortality and unfavourable neurological outcome [defined as death, vegetative, or severely disabled (GOS 1-3)] were built, using as co-variates the percentage of total monitoring time each patient experienced one or more ICP insults associated with worse GOS (indicated on the graph as the red zone), together with the IMPACT model core variables [32,33] (age, admission motor GCS, pupils).

All analyses were done in Matlab 2014b ${ }^{\circledR}$ (The MathWorks, Natick, MA, USA).

\section{Results}

The colour-coded plots visualizing the correlations between GOS at 6 months and the average number of different types of ICP insults are shown in Fig. 2. Two clear overall regions emerge: a predominantly blue zone, indicating types of ICP insults that occur more frequently in patients with higher GOS; and a predominantly red zone, indicating types of ICP insults that occur more frequently in patients with lower GOS. The transition curve between the blue and red zone is approximately exponential: for higher insult intensities, the transition occurs at shorter insult durations and, conversely, for lower insult intensities the transition occurs at longer insult durations. In adults, the duration above which ICP thresholds of 15, 20, 25, and $30 \mathrm{mmHg}$ are associated with worse outcome, is $223,37,12$, and $8 \mathrm{~min}$, respectively. In children, at intensity thresholds of 15 and $20 \mathrm{mmHg}$, the respective transitions occur at shorter durations of 48 and $8 \mathrm{~min}$. The transition curves of the two cohorts are shown together in Fig. 3.

In a multivariate analysis, the percentage of total monitoring time spent by each patient in the red zone was an independent significant predictor of death, both in adults [OR 9.91 (95\% Cl 2.67-36.8); $\mathrm{p}<0 \cdot 001$ ] as in children [OR $\left.178 \times 10^{3}\left(95 \% \mathrm{Cl} 1.02-309 \times 10^{8}\right) ; \mathrm{p}<0 \cdot 050\right]$. The percentage of monitoring time in the red zone was also a significant predictor of unfavourable outcome in adults [OR 3.97 (95\% CI 1.30-12-1); p=0.014] (ESM Tables 2-5). The percentage of monitoring time in the red zone during the first day only was a significant independent predictor of mortality [OR 2.90 (95\% Cl 1.03-8.14); $\mathrm{p}=0.039]$, but not of 6-month unfavourable outcome ([OR $1 \cdot 80$ (95\% Cl 0.78-4.18); $\mathrm{p}=0 \cdot 16]$ in adults. In paediatric TBI patients, there was a trend towards significance for the percentage of monitoring time in the red zone during the first day only, for both mortality [OR $266 \times 10^{1}$ 
$\left.\left(95 \% \mathrm{Cl} 0.564-126 \times 10^{5}\right) ; p=0.062\right]$ and unfavourable outcome [OR $5.82(95 \% \mathrm{Cl}$ 0.861-39.4); $p=0.065]$ (ESM Tables 6-9).

Figure 4 shows the transition curves resulting from visualizations of active and passive autoregulation insults (the corresponding colour-coded plots are available in the ESM Fig. 1, the percentages of active and passive insults in each cohort in the ESM Table 10). In the adult as well as in the paediatric population, the transition curve for passive insults shifts towards the left, and the transition curve for active insults shifts towards the right, with respect to the 'all insults' curve. In adults, episodes above $20 \mathrm{mmHg}$ when cerebrovascular autoregulation is active, had a duration of 233 minutes before they were associated with worse outcome, versus 19 minutes when autoregulation was passive. Duration thresholds derived from the active and passive transition curves for commonly used intensity thresholds can be found in the ESM Table 11.

Finally, the association of ICP insults and outcome was plotted for three different levels of CPP. In adults as well as in children, when CPP was below $50 \mathrm{mmHg}$, almost all ICP insults were associated with worse outcome, regardless of duration and intensity (Fig. 5). When CPP was above $50 \mathrm{mmHg}$, the transition curve shifted to the right as compared to the 'all insults' curve. At CPPs of 60 and $70 \mathrm{mmHg}$ there was a further small rightward shift of the transition curve (Fig. 6).

\section{Discussion}

In this study, the univariate relationship between 6-month neurological outcome and insults of raised ICP is summarized in colour-coded plots. These plots do not represent the cumulative time/pressure dose per patient, but per type of insult, characterized by duration and intensity. The cumulative ICP burden per patient (defined as the percentage of time in the red zone) was assessed through multivariate analysis, and was independently associated with mortality at 6 months.

The main finding is the emergence of clearly distinct insult intensity and duration thresholds that occur more frequently in patients with lower 6-month GOS, delineated by an approximately exponential transition curve. This supports the 'dose of ICP' concept, which has been proposed earlier as the proportion of hourly measurements above $20 \mathrm{mmHg}$ [12], or the area under the curve of hourly values above $20 \mathrm{mmHg}$ [15]. The present study builds further on this concept. First, the use of minute-by-minute monitoring data allows for a 
more precise definition of the concept 'dose'. Second, by considering all intensity thresholds above $10 \mathrm{mmHg}$, the 'dose' is not limited to the $20-\mathrm{mmHg}$ threshold. The time pressure burden was identified for the whole range of ICP's between $15-25 \mathrm{mmHg}$, which is the same range of ICPs that was identified in the early association studies [8-12]. It is striking that the exponential decay transition curve in adults (Fig. 2, left) approximates the vertical 15- $\mathrm{mmHg}$ line, which indicates that insults between 15 and $20 \mathrm{mmHg}$, if sustained for sufficiently long, can already lead to worse outcomes. Above $20 \mathrm{mmHg}$, the vast majority of insults are clearly deleterious with the exception of those lasting less than $37 \mathrm{~min}$ in adults. Above $25 \mathrm{mmHg}$, the association with worse GOS occurs within $12 \mathrm{~min}$. An alternative explanation for the transition curves occurring at lower intensity values for longer durations could have been the presence of patients who underwent decompressive craniectomy: these patients were no longer able to reach high ICP values but nevertheless could have a poor neurological outcome; however, we do not believe that this was the case, because when this clinical group was excluded, visualization results remained unchanged. (ESM Fig. 3 and Tables 9-10).

The transition curve for the paediatric cohort resembles that of adults, but the entire curve has shifted to the left (Fig. 3 and ESM Table 11): episodes of lower intensity and shorter duration than for adults are associated with worse outcomes. In children, the exponential decay transition curve approximates the vertical $10-\mathrm{mmHg}$ line (Fig. 2, right), and above $20 \mathrm{mmHg}$, the association with worse GOS occurs within 8 minutes.

Cerebrovascular pressure autoregulation is often impaired in severe TBI, and such impairment is independently associated with worse outcomes [31, 34-36]. The autoregulatory status has a major effect on the transition curves in both adults and children. When autoregulation is intact, the transition curve shifts to the right. Conversely, with impaired autoregulation, there is a dramatic shift to the left in the transition curves, suggesting a higher vulnerability to raised ICP (Fig. 4; ESM Table 11). This is in line with the findings from a recent retrospective study, where individualized ICP thresholds based on the autoregulatory status of the patient were more predictive for mortality than fixed thresholds of 20 and $25 \mathrm{mmHg}$ [37].

At a CPP below $50 \mathrm{mmHg}$, the plots become almost uniformly red (Fig. 5). In other words, when CPP is critically low, ICP is no longer a univariate predictor for outcome. Caution is warranted when interpreting these data, because arterial blood pressure (ABP) was not always measured and zeroed at the same level: in some patients the right atrium level was used, in some the external auditory meatus, while in some patients this information 
was not available. Therefore, the statements on the influence of CPP on the association between ICP and outcome are only very general, and cannot be used to recommend specific CPP thresholds. In adults, a trend can be observed such that ICP insults in the range $18-23 \mathrm{mmHg}$ can have a longer duration (in the order of minutes) at higher CPPs. When ICP is above $25 \mathrm{mmHg}$, a higher CPP has no effect (Fig. 6, left). In children, the same effect can be observed, albeit at lower ICP values: ICP insults in the range 13-20 mmHg, but not above 20 $\mathrm{mmHg}$, can be tolerated for a slightly longer duration (Fig. 6, right).

This study suffers from several limitations, in addition to the ones already mentioned above. First, the number of patients is relatively small. The creation of large high-resolution databases of good quality in TBI is challenging. Current and future initiatives to create joint databases [38] will facilitate addressing basic research questions such as the relationship between physiological thresholds and outcome. Second, because of their longer total monitoring time, longer-staying patients might have been overrepresented, possibly introducing bias. However, when using daily averages of the number of insults, instead of the entire monitoring time, all visualization results remain unchanged. (ESM Fig. 2). Third, the analysis has been done in treated cohorts of patients. In retrospect, it is not possible to unravel the individual contributions on clinical outcome of the intensity of secondary brain injury insults versus the possible benefit or harm of the medical interventions employed to manage them. Fourth, the patient data collection extended over 10 years, and the critical care management of TBI might have changed substantially over this period. We believe the results are robust over potential differences in management, because when plotting the graphs in different subgroups of patients from different time eras, the approximately exponential transition curve remained (data not shown). Fourth, only the obvious artefacts were removed, via visual inspection by two independent experts. Data was collected in a prospective way, and at the time of collection, attention was paid to avoid incorrect data entries as much as possible. However, this does not exclude that some less obvious artefacts might still have been wrongfully identified as an ICP insult, and used in the analysis. Automatic artefact removal algorithms were not used, because we wanted to retain as much of the granularity of the minute-by-minute data as possible, and because we presumed that the incidence of erroneous entries was low, in proportion to the large total amount of insults analysed (8181337 insults in adults, and 2586632 insults in children, ESM table 10). Fifth, the observed associations, visualized in a single clear image, do not imply a causative relationship between the insults and the outcome, and other unmeasured confounders might have played a role. 
The present study is an attempt to add to the current knowledge in developing a new concept of 'pressure and time burden' for intracranial hypertension. The results indicate that the ICP threshold in TBI patients depends on insult duration, age, CPP, and the autoregulatory status. These findings need to be confirmed in other data sets. These data visualizations are not intended to issue a new direct recommendation on specific ICP thresholds: they are meant as exploratory tools. On the basis of our findings, perhaps an argument could be made that ICPs exceeding $25 \mathrm{mmHg}$ in adults, and $20 \mathrm{mmHg}$ in children, are absolute emergencies which could potentially lead to secondary brain injury in a couple of minutes, and may thus be more appropriate clinical triggers to initiate aggressive therapy than the more commonly used value of $20 \mathrm{mmHg}$. This might also explain why previous randomized controlled trials in TBI patients where a 20-mmHg static threshold was used [19-21] were destined to fail. In addition, ICPs in the $15-$ to $20-\mathrm{mmHg}$ range in adults, or $10-15 \mathrm{mmHg}$ in children, are not to be considered harmless. Additional information through clinical examination, multimodality monitoring, imaging, and assessment of autoregulation might be considered with pressures in that range, in order to estimate the risk for secondary brain injury and the need for aggressive ICP-lowering treatments [39].

\section{Conclusion}

An image can be worth a thousand words: the visualizations presented here summarize the complexity and dynamic aspect of secondary insults of raised ICP in TBI, showing that not all TBI patients are equal in their ability to cope with such injury.

\section{Acknowledgments}

We wish to acknowledge the non-co-author members of the Brain-IT steering group: Barbara Gregson, Tim Howells, Karl Kiening, Julia Mattern, Arminas Ragauskas and Juan Sahuquillo, for collecting data and granting permission through the steering group to use them for this project. Similarly, we would also like to acknowledge the contributors to the original study resulting in the paediatric database used here: R.J. Forsyth, B. Fulton, P.J.D. Andrews, A.D. Mendelow, and R.A. Minns.

The present study was supported by the Foundation for Scientific Research Flanders (FWO) (Research project G. 0904.11). Geert Meyfroidt receives funding from FWO as senior clinical investigator (1846113N). Greet Van 
den Berghe receives long-term structural research financing via the Methusalem program funded by the Flemish Government (METH/08/07). BrainlT was funded by the European Framework Programme (FP5-QLRI2000-00454, QLGT-2002-00160 and FP7-IST-2007-217049). The NEMO project in the University Hospital Edegem (Antwerp), Belgium was funded by the Flemish Government Agency for Innovation by Science and Technology (IWT) - Applied Biomedical Research (TBM) program.

\section{Contributors}

FG, BDP, GVdB and GM conceived and designed the study. FG developed the visualization methodology; GM and BDP supervised the design and the interpretation of the plots. FG and GM drafted the final manuscript. $B D P, G M, I C, G C, P J, B F, A M, P E, P N, M S, T Y M L, K J$ collected patient data. BDP, IP, IC, GC, PE, PN, RD, LM, and IC are members of the Brain-IT steering group. All authors participated in discussions that lead to the design of the study, and have read and approved the final manuscript. The sponsors of the study had no input in study design, data collection, data analysis, data interpretation, writing of the report, or the decision to submit the paper for publication. The corresponding author had full access to all data in the study and had final responsibility for decision to submit for publication. 
References:

1. Hydera AA, Wunderlich CA, Puvanachandra P, Gururaj G, Kobusingye OC (2007) The impact of traumatic brain injuries: A global perspective. NeuroRehabilitation 22:341-353.

2. Stein SC, Georgoff P, Meghan S, Mizra K, Sonnad SS (2010) 150 years of treating severe traumatic brain injury: a systematic review of progress in mortality. J Neurotrauma 27:1343-53.

3. Patel HC, Bouamra O, Woodford M, King AT, Yates DW, Lecky FE (2005) Trends in head injury outcome from 1989 to 2003 and the effect of neurosurgical care: an observational study. Lancet 366:15381544.

4. Rosenfeld JV, Cooper DJ (2005) Management of severe head injury: can we do better? Lancet 366:1509-1510.

5. Guillaume J, Janny P (1951) Continuous intracranial manometry; importance of the method and first results. Rev Neurol (Paris) 84:131-42.

6. Lundberg N, Troupp $\mathrm{H}$, Lorin $\mathrm{H}$ (1965) Continuous recording of the ventricular-fluid pressure in patients with severe acute traumatic brain injury. A preliminary report. J Neurosurg 22(6):581-590.

7. Brain Trauma Foundation, American Association of Neurological Surgeons (AANS), Congress of Neurological Surgeons (CNS), AANS/CNS Joint Section on Neurotrauma and Critical Care (2007) Guidelines for the management of severe traumatic brain injury. VI. Indications for intracranial pressure monitoring. J Neurotrauma 24 (Suppl 1):S37-S44.

8. Marshall LF, Smith RW, Shapiro HM (1979) The outcome with aggressive treatment in severe head injuries. Part II: acute and chronic barbiturate administration in the management of head injury. J Neurosurg 50(1):26-30.

9. Narayan RK, Kishore PR, Becker DP, Ward JD, Enas GG, Greenberg RP, Domingues Da Silva A, Lipper MH, Choi SC, Mayhall CG, Lutz HA 3rd, Young HF (1982) Intracranial pressure: to monitor or not to monitor? A review of our experience with severe head injury. J Neurosurg 56(5):650-659.

10. Saul TG, Ducker TB (1982) Effect of intracranial pressure monitoring and aggressive treatment on mortality in severe head injury. J Neurosurg 56(4):498-503.

11. Eisenberg HM, Frankowski RF, Contant CF, Marshall LF, Walker MD (1988) High-dose barbiturate control of elevated intracranial pressure in patients with severe head injury. J Neurosurg 69(1):15-23.

12. Marmarou A, Anderson RL, Ward JD, Choi SC, Young HF, Eisenberg HM, Foulkes MA, Marshall LF, Jane JA (1991) Impact of ICP instability and hypotension on outcome in patients with severe head trauma. J Neurosurg 75:S59-S66.

13. Badri S, Chen J, Barber J, Temkin NR, Dikmen SS, Chesnut RM, Deem S, Yanez ND, Treggiari MM (2012) Mortality and long-term functional outcome associated with intracranial pressure after traumatic brain injury. Intensive Care Med 38(11):1800-1809.

14. Sigurtà A, Zanaboni C, Canavesi K, Citerio G, Beretta L, Stocchetti N (2013) Intensive care for pediatric traumatic brain injury. Intensive Care Med 39(1):129-136.

15. Vik A, Nag T, Fredriksli OA, Skandsen T, Moen KG, Schirmer-Mikalsen K, Manley GT (2008) Relationship of "dose" of intracranial hypertension to outcome in severe traumatic brain injury. J Neurosurg 109:678-684.

16. Kahraman S, Dutton R, Hu P, Xiao Y, Aarabi B, Stein DM, Scalea TM (2010) Automated measurement of "pressure times time dose" of intracranial hypertension best predicts outcome after severe traumatic brain injury. J Trauma 69:110-118.

17. Chambers IR, Jones PA, Lo TY, Forsyth RJ, Fulton B, Andrews PJ, Mendelow AD, Minns RA (2006) Critical thresholds of intracranial pressure and cerebral perfusion pressure related to age in paediatric head injury. J Neurol Neurosurg Psychiatry 77(2):234-240.

18. Adelson PD, Bratton SL, Carney NA, Chesnut RM, du Coudray HE, Goldstein B, Kochanek PM, Miller HC, Partington MP, Selden NR, Warden CR, Wright DW (2003) Guidelines for the acute medical management of severe traumatic brain injury in infants, children and adolescents. Pediatr Crit Care Med 4(3):S72-75. 
19. Cremer OL, van Dijk GW, van Wensen E, Brekelmans GJ, Moons KG, Leenen LP, Kalkman CJ (2005) Effect of intracranial pressure monitoring and targeted intensive care on functional outcome after severe head injury. Crit Care Med 33(10):2207-2213.

20. Cooper DJ, Rosenfeld JV, Murray L, Arabi YM, Davies AR, D'Urso P, Kossmann T, Ponsford J, Seppelt I, Reilly P, Wolfe R, DECRA Trial Investigators, Australian and New Zealand Intensive Care Society Clinical Trials Group (2011) Decompressive craniectomy in diffuse traumatic brain injury. N Engl J Med 364:1493-1502.

21. Chesnut RM, Temkin N, Carney N, Dikmen S, Rondina C, Videtta W, Petroni G, Lujan S, Pridgeon J, Barber J, Machamer J, Chaddock K, Celix JM, Cherner M, Hendrix T (2012) A trial of intracranialpressure monitoring in traumatic brain injury. N Engl J Med 367:2471-2481.

22. Le Roux P (2014) Intracranial pressure after the BEST TRIP trial: a call for more monitoring. Curr Opin Crit Care 20:141-147.

23. Stocchetti N, Maas Al (2014) Traumatic intracranial hypertension. N Eng J Med 370:2121-2130.

24. Le Roux P, Menon DK, Citerio G, Vespa P, Bader MK, Brophy GM, Diringer MN, Stocchetti N, Videtta W, Armonda R, Badjatia N, Böesel J, Chesnut R, Chou S, Claassen J, Czosnyka M, De Georgia M, Figaji A, Fugate J, Helbok R, Horowitz D, Hutchinson P, Kumar M, McNett M, Miller C, Naidech A, Oddo M, Olson D, O'Phelan K, Provencio JJ, Puppo C, Riker R, Robertson C, Schmidt M, Taccone F (2014) Consensus summary statement of the International Multidisciplinary Consensus Conference on Multimodality Monitoring in Neurocritical Care: a statement for healthcare professionals from the Neurocritical Care Society and the European Society of Intensive Care Medicine. Intensive Care Med. 40(9):1189-1209.

25. Güiza F, Depreitere B, Piper I, Van den Berghe G, Meyfroidt G (2014) New look at the $20 \mathrm{mmHg}$ ICP threshold. Critical Care 18 (Suppl 1):P458.

26. Güiza F, Depreitere B, Lo TY, Jones PA, Chambers IR, Van den Berghe G, Meyfroidt G (2014) Visualizing secondary insults of elevated ICP in pediatric TBI. Intensive Care Med 40 (Suppl 1):S18;0037.

27. Piper I, Citerio G, Chambers I, Contant C, Enblad P, Fiddes H, Howells T, Kiening K, Nilsson P, Yau YH (2003) The Brain-IT group: concept and core dataset definition. Acta Neurochir (Wien) 145: 615-628.

28. Enblad P, Nilsson P, Chambers I, Citerio G, Fiddes H, Howells T, Kiening K, Ragauskas A, Sahuquillo J, Yau YH (2004) R3-survey of traumatic brain injury management in European Brain IT centres year 2001. Intensive Care Med 30(6):1058-1065.

29. Feyen BFE, Sener S, Jorens PG, Menovsky T, Maas AI (2012) Neuromonitoring in traumatic brain injury. Minerva Anestesiol 78:949-958.

30. Nilsson P, Enblad P, Chambers I (2005) Survey of traumatic brain injury management in European Brain-IT centres year 2001. Acta Neurochir (Suppl) 95:51-53.

31. Depreitere B, Güiza F, Van den Berghe G, Schuhmann MU, Maier G, Piper I, Meyfroidt G (2014) Pressure autoregulation monitoring and cerebral perfusion pressure target recommendation in severe traumatic brain injury patients based on minute-by-minute monitoring data. J Neurosurg 120:14511457.

32. Steyerberg EW, Mushkudiani N, Perel P, Butcher I, Lu J, McHugh GS, Murray GD, Marmarou A, Roberts I, Habbema JD, Maas Al (2008) Predicting outcome after traumatic brain injury: development and international validation of prognostic scores based on admission characteristics. PLoS Med. 5(8):e165.

33. IMPACT: International Mission for Prognosis and Analysis of Clinical Trials in TBI (2015) http://www.tbi-impact.org/ Accessed 11 March 2015.

34. Sviri GE, Aaslid R, Douville CM, Moore A, Newell DW (2009) Time course for autoregulation recovery following severe traumatic brain injury. J Neurosurg 111:695-700.

35. Czosnyka M, Smielewski P, Kirkpatrick P, Laing RJ, Menon D, Pickard JD (1997) Continuous assessment of the cerebral vasomotor reactivity in head injury. Neurosurgery 41:11-19.

36. Jaeger M, Schuhmann MU, Soehle M, Meixensberger J (2006) Continuous assessment of cerebrovascular autoregulation after traumatic brain injury using brain tissue oxygen pressure reactivity. Crit Care Med 34(6):1783-1788 
37. Lazaridis C, DeSantis SM, Smielewski P Menon DK, Hutchinson P, Pickard JD, Czosnyka M (2014) Patient-specific thresholds of intracranial pressure in severe traumatic brain injury. J Neurosurg 120:893-900.

38. CENTER-TBI (2015) https://www.center-tbi.eu/ Accessed 6 March 2015

39. Chesnut RM (2015) What the BEST TRIP study means to me as the principle investigator. https://www.braintrauma.org/dr-chesnut-commentary/ Accessed 24 March 2015 


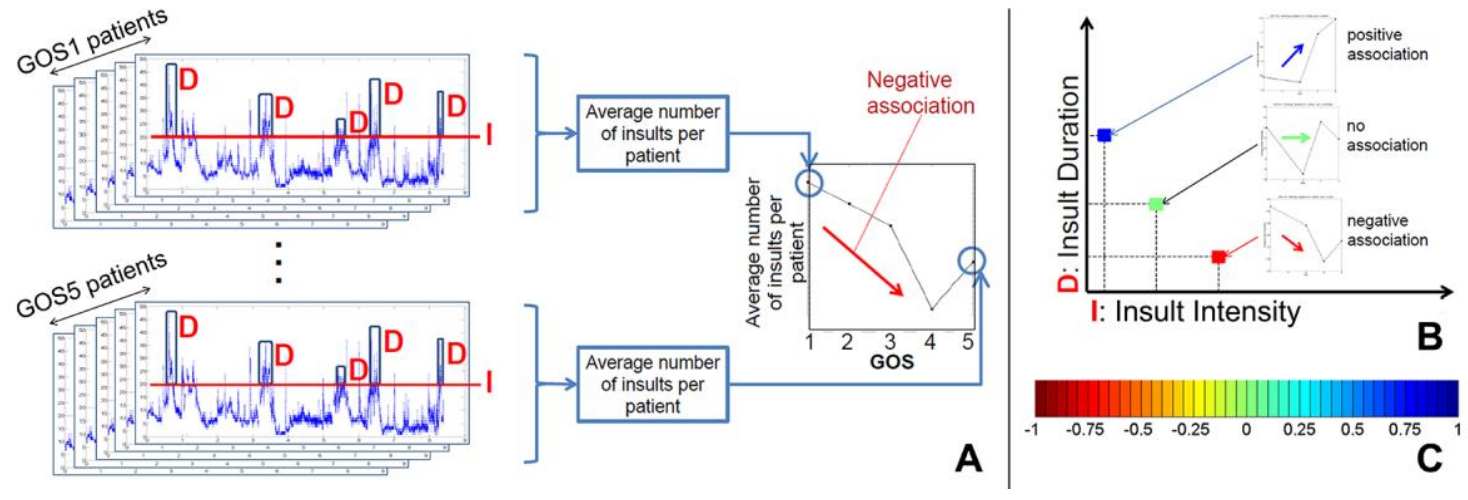

Fig. 1 Visualization methodology. The average number of insults of ICP above an intensity threshold I (in $\mathrm{mmHg}$ ), lasting for at least a duration threshold $\boldsymbol{D}$ (in minutes) is computed for each GOS category and the Pearson correlation is computed (a). The correlation between GOS and the average number of insults per GOS category is computed for all $\boldsymbol{I}$ and $\boldsymbol{D}$ combinations $(\mathbf{b})$ and given a colour according to a predefined colour map ranging from -1 in dark red to +1 in dark blue (c). 

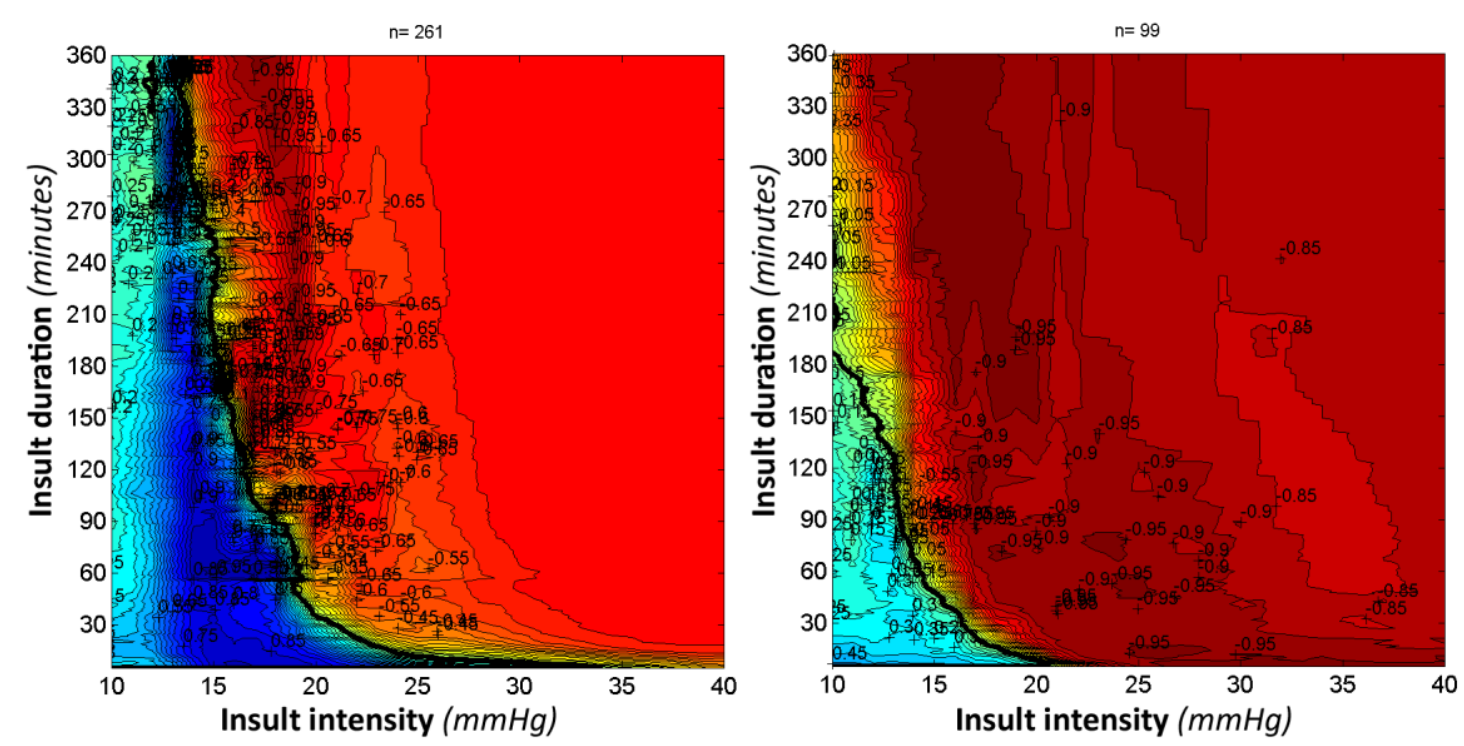

Fig. 2 Visualization of correlation between GOS and average number of ICP insults per GOS category. Left adult cohort ( $n=261$ ). Right paediatric cohort $(n=99)$. Each colour-coded point in the graph refers to a number of episodes of ICP, defined by a certain ICP intensity threshold (X-axis), and a certain duration threshold (Y-axis). Such an episode is called an ICP insult. The univariate correlation of each type of ICP insult (characterized by ICP intensity and duration thresholds) with outcome is colour-coded according to the scale in Fig. 1c. Dark red episodes mean that such ICP insults, on average, are associated with worse outcome (lower GOS categories); dark blue episodes mean that such ICP insults, on average, are associated with better outcome (higher GOS categories). The contour of zero correlation is highlighted in black, and is called the transition curve. 


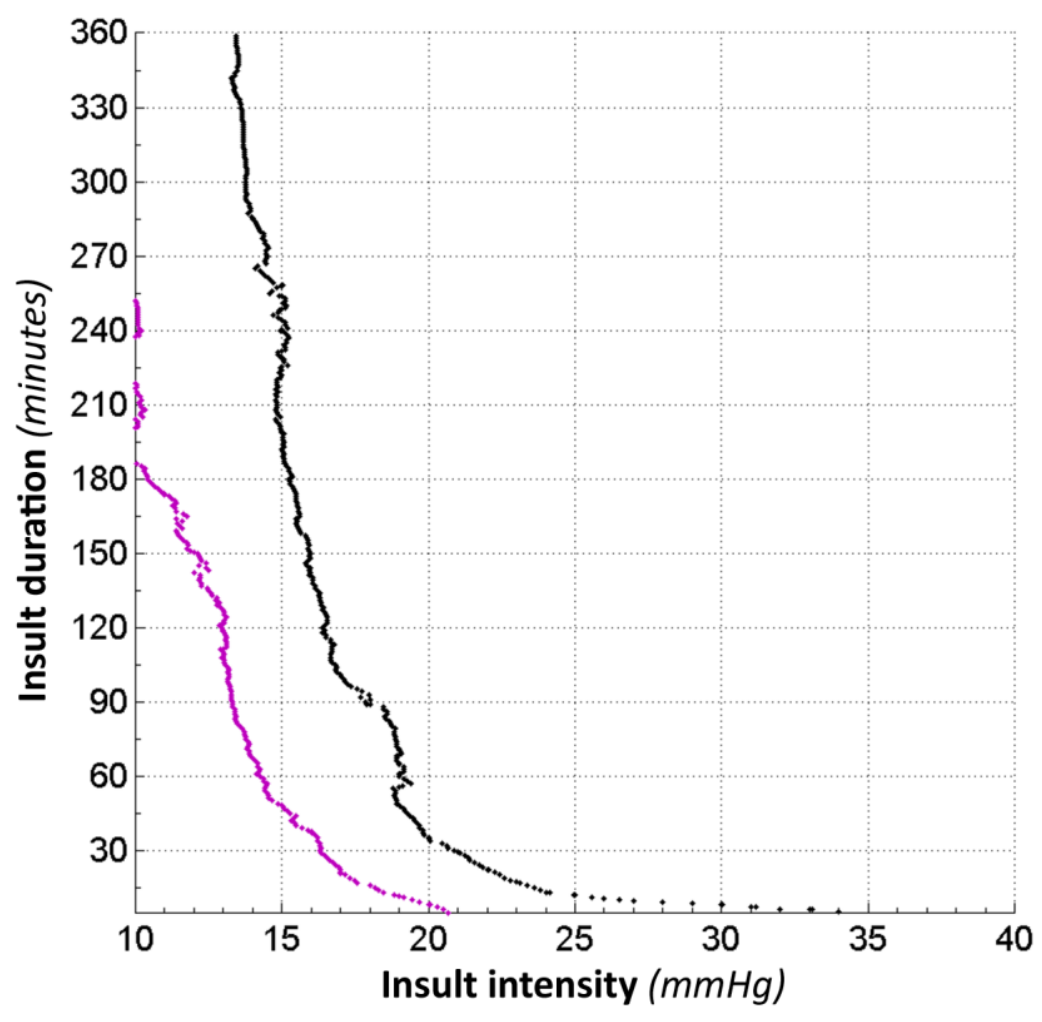

Fig. 3 Comparison of transition curves in adult (black) and paediatric (purple) cohorts. Transition curves are the lines of zero correlation between ICP insults and outcome. Episodes above and to the right of this transition curve are associated with worse outcomes. 

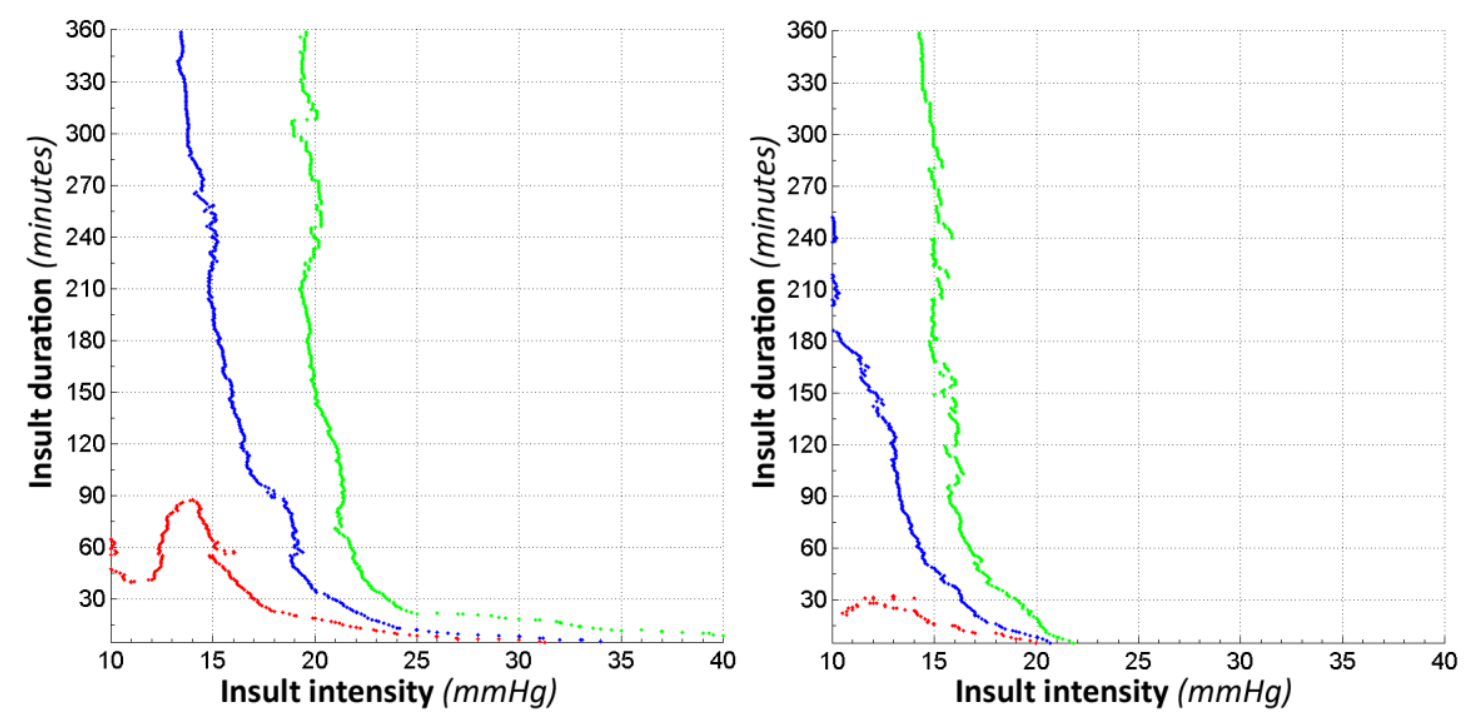

Fig. 4 Transition curve visualizations according to cerebrovascular autoregulatory status. The transition curves for all insults (irrespective of autoregulation) are drawn in blue, while the transition curves for autoregulation active insults are shown in green and for autoregulation passive insults in red. Left adult cohort $(n=261)$. Right paediatric cohort ( $n=99$ ). Transition curves are the lines of zero correlation between ICP insults and outcome. Episodes above and to the right of this transition curve are associated with worse outcomes. 

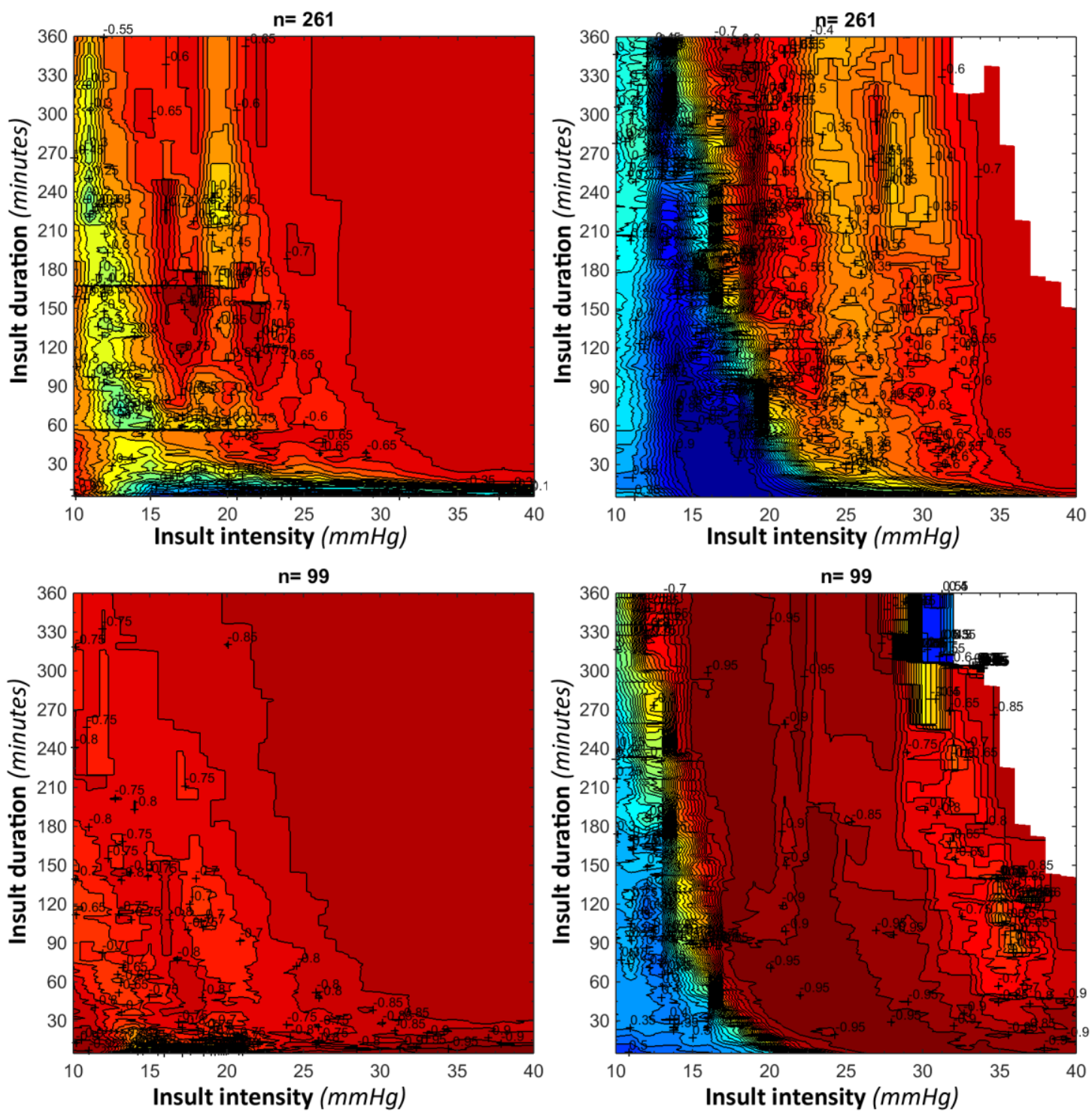

Fig. 5 Visualization of correlation between GOS and average number of ICP insults per GOS category according to a cerebral perfusion pressure (CPP) threshold of $50 \mathrm{mmHg}$. For an explanation of this colour-coded plot, refer to the legend of Fig, 1. Left panel CPP $\leq 50 \mathrm{mmHg}$. Right panel CPP $>50 \mathrm{mmHg}$. Above adult cohort $(n=261)$. Below paediatric cohort $(n=99)$. 

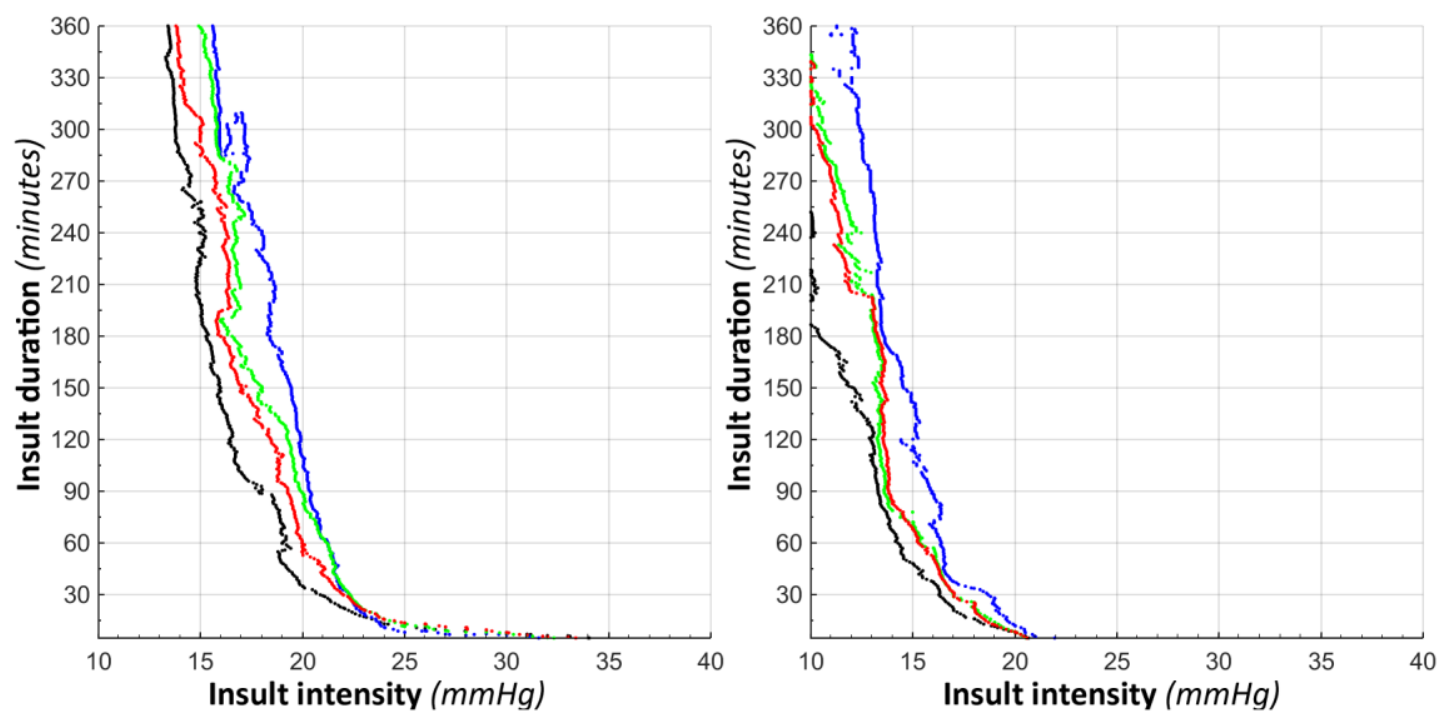

Fig. 6 Transition curves of ICP insults according to three different thresholds of cerebral perfusion pressure (CPP). For an explanation on transition curves, refer to the legend of Fig. 4. All insults irrespective of CPP (black); insults with CPP > $50 \mathrm{mmHg}$ (red); with CPP > $60 \mathrm{mmHg}$ (green) and with CPP > $70 \mathrm{mmHg}$ (blue). Left panel adult cohort $(\mathrm{n}=261)$. Right panel paediatric cohort $(\mathrm{n}=99)$. 